\section{Quality of care in adult patients with inflammatory bowel disease transferring between healthcare providers: multicentre audit}

There is a paucity of evidence regarding patients with IBD who need to transfer their care to a new secondary or tertiary care provider, both in terms of how effectively this is done and whether it affects outcome. This has important implications for transition from paediatric to adult services but also, with an increasingly mobile population, for adult patients who relocate for university, work, social or other reasons. In this issue the GLINT Research Network report a multicentre audit (154 cases, 16 hospitals, London). More than 50\% of transfers were for relocation with other reasons including referral to the private sector, seeking a second opinion, referral for a specialist opinion. Most onward referrals were from primary care despite previous follow-up being with a secondary care provider. Information included was incomplete in more than $70 \%$ - including details of diagnosis, treatment (including surgical) and the results of the most recent investigation. The authors advocate that improved communication between the patient, gastroenterologists and primary care is essential to facilitate smooth transfer of care including a summary of the patient's assessments and treatment. The increasing use of digital health technologies and information sharing may enable patients to take individual ownership of the transfer process. There is a powerful accompanying commentary from Phillip Smith, Associate Editor for Frontline Gastroenterology and patient with Crohn's disease - Moving house - moving medical teams: keeping control of my IBD (See page 5).

\section{Getting to grips with sarcopenia: recent advances and practical management for the gastroenterologist}

Sarcopenia is a progressive and generalised disorder of skeletal muscle strength, function and mass that is most commonly associated with the normal ageing process although is increasingly recognised in inflammatory bowel disease and liver disease. In this edition Hollingworth and colleagues discuss the recent advances and practical management. Sarcopenia is common but poorly recognised by clinicians. Pathogenesis is multifactorial and sarcopenic obesity well described. The authors discuss sarcopenic obesity, cachexia, and frailty as clinically distinct but overlapping conditions. The SARC-F (see table 2 in the paper) is a useful screen for symptomatic sarcopenia assessing strength, difficulties with mobility (rise from chair, climbing stairs) and number of falls in the last year. Diagnosis is by grip strength, dual energy X-ray absorptiometry, gait speed and CT measurement of muscle mass. Treatment is nutritional intervention (particularly protein intake) and by resistance exercise programmes. There are no proven pharmacological treatments although treatment of underlying conditions is important. There is much ongoing research in this area but in summary better recognition and management is likely to improve outcomes for patients with underlying gut and liver disease. Essential reading (See page 53).

\section{Systematic review: the impact of inflammatory bowel disease- related fatigue on health- related quality of life}

Fatigue is frequently in inflammatory bowel disease and impacts on health related quality of life (HRQoL). In this issue Radford and colleagues systematically review the literature. Eleven studies were included (2823 participants). Fatigue was significantly related to three HRQoL linked themes - symptom acceptance and management, psychosocial well-being and management and physical activity. Physical activity was impaired by higher fatigue levels, lowering HRQoL, but it was also used as a means of reducing fatigue and improving HRQoL. The authors conclude that better understanding of the impact of IBD fatigue has the potential to improve HRQoL, reduce costs and allow patients to feel a sense of control through effective selfmanagement. Important in a condition where chronic symptoms and treatment escalation is common (See page 11).

\section{Twitter debate: controversies in the management of portal hypertension}

I hope you all enjoy the monthly Frontline Gastroenterology Twitter debates - usually around published content - and popular with great engagement (and reach). In this issue Chun and colleagues summarise the discussion around controversies in the management of portal hypertension from the September 2019 debate. Key points - all endoscopists on the bleeder rota should know how to manage variceal bleeding, antibiotics will reduce mortality, avoid over transfusion, ensure patients are adequately resuscitated prior to starting vasoactive agents, intubate prior to endoscopy, band ligation is the treatment of choice for oesophageal varices, gastric varices may require glue injection. Read the well referenced report for the detail. Great CPD. Look out for future debates and if you have ideas for content/want to get involved make contact with the editorial team (See page 77).

\section{Guideline review: British Society of Gastroenterology/UK-PSC guidelines for the diagnosis and management of primary sclerosing cholangitis}

In an excellent review of the British Society of Gastroenterology guidelines for the diagnosis and management of primary sclerosing cholangitis (PSC) ${ }^{1}$ Nicoletti and colleagues discuss the key issues. Estimated incidence is around 1 in 100000 . There is no effective pharmacological treatment and the potential to progress to chronic liver disease with PSC accounting for about $15 \%$ of adult liver transplants. The clinical features and diagnosis are discussed in detail. More than $80 \%$ also have inflammatory bowel disease. All patients require lifelong follow-up including disease surveillance and active management of specific symptoms (see table 2 in the paper). Multidisciplinary input is essential especially when specific interventions such as endoscopic management of biliary strictures is being considered. Liver transplant is the only treatment able to modify the natural history of the disease. Great summary of important guidance (See page 62). 


\section{Finally}

Please let us know if there is a recent guideline you would like to review - see instructions for authors for the specific requirements. The intention is to highlight and discuss the main points and in doing so signpost readers to useful national and international guidance that will help us with our day to day clinical practice and so help us manage patients better.
Please enjoy this issue. Please continue to read, enjoy, and feedback on the journal. Follow us on twitter@FrontGastro_BMJ and listen to our regular podcasts accessed via the journal website https://fg.bmj.com/

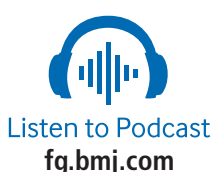

ORCID ID

R Mark Beattie http://orcid.org/00000003-4721-0577

\section{REFERENCE}

1 Chapman MH, Thorburn D, Hirschfield GM, et al. British Society of gastroenterology and UK-PSC guidelines for the diagnosis and management of primary sclerosing cholangitis. Gut 2019;68:1356-78. 